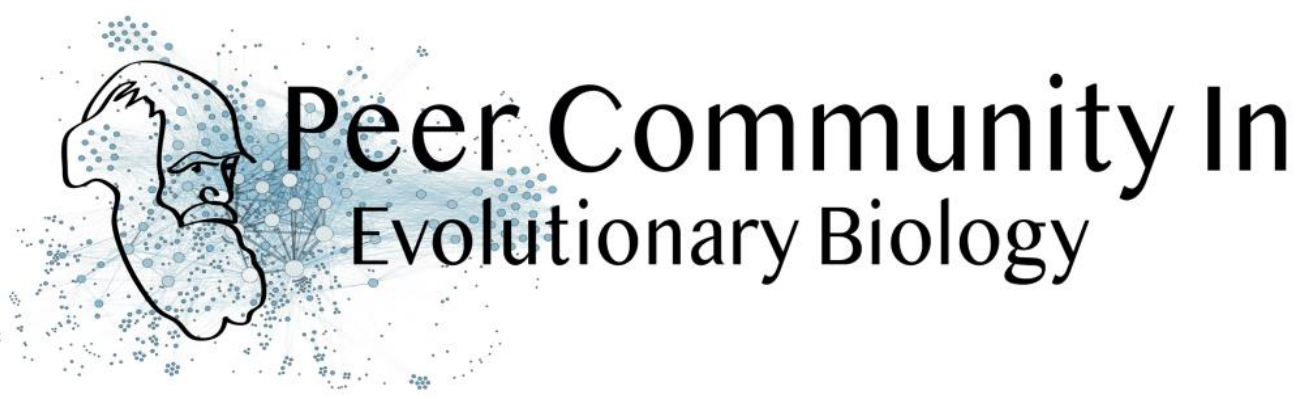

\title{
What makes a parasite successful? Parasitoid wasp venoms evolve rapidly in a host-specific manner
}

\section{Élio Sucena}

based on reviews by Simon Fellous, Alexandre Leitão and one anonymous reviewer

\section{A recommendation of:}

Parasitic success and venom composition evolve upon specialization of parasitoid wasps to different host species

Fanny Cavigliasso, Hugo Mathé-Hubert, Jean-Luc Gatti, Dominique Colinet, Marylène Poirié(2021), bioRxiv, 2020.10.24.353417, ver. 3 peer-reviewed and recommended by Peer Community In Evolutionary Biology.

\section{Open Access https://doi.org/10.1101/2020.10.24.353417}

Submitted: 26 October 2020, Recommended: 04 March 2021

Published: 14 April 2021

Copyright: This work is licensed under the Creative Commons Attribution-NoDerivatives 4.0 International License. To view a copy of this license, visit http://creativecommons.org/licen ses/by-nd/4.0/
Parasitoid wasps have developed different mechanisms to increase their parasitic success, usually at the expense of host survival (Fellowes and Godfray, 2000). Eggs of these insects are deposited inside the juvenile stages of their hosts, which in turn deploy several immune response strategies to eliminate or disable them (Yang et al., 2020). Drosophila melanogaster protects itself against parasitoid attacks through the production of specific elongated haemocytes called lamellocytes which form a capsule around the invading parasite (Lavine and Strand, 2002; Rizki and Rizki, 1992) and the subsequent activation of the phenoloxidase cascade leading to the release of toxic radicals (Nappi et al., 1995). On the parasitoid side, robust responses have evolved to evade host immune defenses as for example the Drosophila-specific endoparasite Leptopilina boulardi, which releases venom during oviposition that modifies host behaviour (Varaldi et al., 2006) and inhibits encapsulation (Gueguen et al., 2011; Martinez et al., 2012).

Studies have shown that the wasp parasitic capacity is correlated to venom presence and its content (Colinet et al., 2009; Poirié et al., 2014), including that evolution of venom protein composition is driven by different levels of host susceptibility to infection (Cavigliasso et al., 2019). However, it had not been determined to this day, if and how parasitic range can affect venom protein composition and to which extent host specialization requires broad-spectrum factors or a plethora of specialized components.

These outstanding questions are now approached in a study by Cavigliasso and colleagues (Cavigliasso et al., 2021), where they perform experimental evolution of L. boulardi for 9 generations exposing it to different Drosophila host species and genetic backgrounds (two strains of D. melanogaster, D. simulans and D. yakuba). The authors tested whether the parasitic success of each selection 


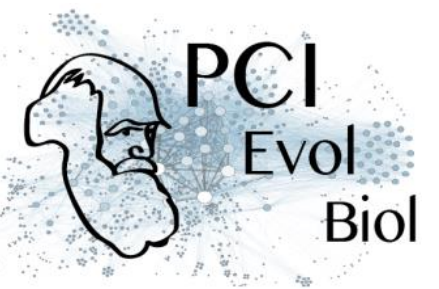

regime was host-specific and how they influenced venom composition in parasitoids. For the first part, infection outcomes were assayed for each selection regime when cross-infecting different hosts. To get a finer measurement of the mechanisms under selection, the authors differentiated three phenotypes: overall parasitic success, encapsulation inhibition and escape from capsule. Throughout the course of experimental evolution, only encapsulation inhibition did not show an improved response upon selection on any host. Importantly, the cross-infection scenario revealed a clear specificity to the selected host for each evolved resistance.

As for venom composition, a trend of differential evolution was detected between host species, although a significant part of that was due to a larger differentiation in the D. yakuba regime, which showed a completely different directionality. Importantly, the authors could identify some of the specific proteins targeted by the several selection regimes, whether selected or counter-selected for. Interestingly, the D. yakuba regime is the only case where the key parasitoid protein LbSPNy (Colinet et al., 2009) was not counter-selected and the only regime in which the overall venom composition did not evolve towards the Ism strain, one of the two ancestral strains of L. boulardi used in the study. It is possible that these two results are correlated, since LbSPNy has been described to inhibit activation of the phenoloxidase cascade in D. yakuba and is one of the most abundant proteins in the ISy venom, making it a good target for selection (Colinet et al., 2013). The authors also discuss the possibility that this difference is related to the geographical distribution of the strains of L. boulardi, since each coincide with either D. melanogaster or D. yakuba.

This methodologically broad work by Cavigliasso and colleagues constitutes an important experimental contribution towards the understanding of how parasitoid adaptation to specific hosts is achieved at different phenotypic and mechanistic levels. It provides compelling evidence that venom composition evolves differently in response to specific parasitic ranges, particularly considering the evolutionary difference between the selective hosts. In line with this result, it is also concluded that the majority of venom proteins selected are lineage-specific, although a few broad-spectrum factors could also be detected.

The question of whether parasitic range can affect venom composition and parasitic success is still open to more contributions. A potentially interesting long-term direction will be to use a similar setup of experimental evolution on the generalist L. heterotoma (Schlenke et al., 2007) . On a more immediate horizon, comparing the venom evolution of both $L$. heterotoma and $L$. boulardi under selection with different hosts and under cross-infection scenarios could reveal interesting patterns. The recent sequencing of the $L$. boulardi genome together with the vast number of studies addressing mechanisms of Drosophila resistance to parasitoid infection, will enable the thorough characterization of the genetic basis of host-parasitoid interactions and the deeper understanding of these ubiquitous and economically-relevant relationships.

${ }^{*}$ This recommendation text has been co-written with Tânia F. Paulo who is not a recommender of PCl Evol Biol

\section{References}

Cavigliasso, F., Mathé-Hubert, H., Gatti, J.-L., Colinet, D. and Poirié, M. (2021) Parasitic success and venom composition evolve upon specialization of parasitoid wasps to different host species. bioRxiv, 2020.10.24.353417, ver. 3 peer-reviewed and recommended by Peer Community in Evolutionary Biology. https://doi.org/10.1101/2020.10.24.353417

Cavigliasso, F., Mathé-Hubert, H., Kremmer, L., Rebuf, C., Gatti, J.-L., Malausa, T., Colinet, D., Poiré, M. and Léne. (2019). Rapid and Differential Evolution of the Venom Composition of a Parasitoid Wasp Depending on the Host Strain. Toxins,

11(629). https://doi.org/10.3390/toxins11110629 


\section{$\mathrm{PCl}^{\mathrm{PCl}}$

Colinet, D., Deleury, E., Anselme, C., Cazes, D., Poulain, J., Azema-Dossat, C., Belghazi, M., Gatti, J. L. and Poirié, M. (2013). Extensive inter- and intraspecific venom variation in closely related parasites targeting the same host: The case of Leptopilina parasitoids of Drosophila. Insect Biochemistry and Molecular Biology, 43(7), 601-611. https://doi.org/10.1016/j. ibmb.2013.03.010

Colinet, D., Dubuffet, A., Cazes, D., Moreau, S., Drezen, J. M. and Poirié, M. (2009). A serpin from the parasitoid wasp Leptopilina boulardi targets the Drosophila phenoloxidase cascade.

Developmental and Comparative Immunology, 33(5), 681-

689. https://doi.org/10.1016/j.dci.2008.11.013

Fellowes, M. D. E. and Godfray, H. C. J. (2000). The evolutionary ecology of resistance to parasitoids by Drosophila. Heredity, 84(1), 1-8. https://doi.org/10.1046/j.1365-2540.2000.00685.x

Gueguen, G., Rajwani, R., Paddibhatla, I., Morales, J. and Govind, S. (2011). VLPs of Leptopilina boulardi share biogenesis and overall stellate morphology with VLPS of the heterotoma clade. Virus Research, 160(1-2), 159-165. https://doi.org/10.1016/j.virusres.2011.06.005

Lavine, M. D. and Strand, M. R. (2002). Insect hemocytes and their role in immunity. Insect Biochemistry and Molecular Biology, 32(10), 1295-1309. https://doi.org/10.1016/S09651748(02)00092-9

Martinez, J., Duplouy, A., Woolfit, M., Vavre, F., O'Neill, S. L. and Varaldi, J. (2012). Influence of the virus LbFV and of Wolbachia in a host-parasitoid interaction. PloS One, 7(4), e35081. https://doi.org/10.1371/journal.pone.0035081

Nappi, A. J., Vass, E., Frey, F. and Carton, Y. (1995). Superoxide anion generation in Drosophila during melanotic encapsulation of parasites. European Journal of Cell Biology, 68(4), 450-456.

Poirié, M., Colinet, D. and Gatti, J. L. (2014). Insights into function and evolution of parasitoid wasp venoms. Current Opinion in Insect Science, 6, 52-60. https://doi.org/10.1016/j.cois.2014.10.004

Rizki, T. M. and Rizki, R. M. (1992). Lamellocyte differentiation in Drosophila larvae parasitized by Leptopilina. Developmental and Comparative Immunology, 16(2-3), 103-

110. https://doi.org/10.1016/0145-305X(92)90011-Z

Schlenke, T. A., Morales, J., Govind, S. and Clark, A. G. (2007). Contrasting infection strategies in generalist and specialist wasp parasitoids of Drosophila melanogaster. PLoS Pathogens, 3(10), 1486-1501. https://doi.org/10.1371/journal.ppat.0030158

Varaldi, J., Petit, S., Boulétreau, M. and Fleury, F. (2006). The virus infecting the parasitoid Leptopilina boulardi exerts a specific action on superparasitism behaviour. Parasitology, 132(Pt 6), 747-756. https://doi.org/10.1017/S0031182006009930

Yang, L., Qiu, L., Fang, Q., Stanley, D. W. and Gong-Yin, Y. (2020). Cellular and humoral immune interactions between Drosophila and its parasitoids. Insect Science. https://doi.org/10.1111/17447917.12863 


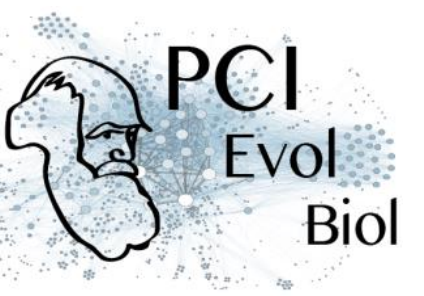

\section{Reviews}

Toggle reviews

\section{Revision round \#1}

2020-12-10

\section{Author's Reply}

Download author's reply (PDF file)Download tracked changes file

Dear recommender and reviewers, we thank you for all your comments and advice. Attached you will find our responses and a version of the manuscript with the track change to follow our edits. The line number in our responses correspond to the number in version without track change (so the "real" line number). Best regards

\section{Decision round \#1}

This manuscript by Cavigliasso, Colinet, Poirié and co-workers, tests the relationship between the evolution of parasitic success and venom composition in L. boulardi and several Drosophila hosts. It combines a wide range of techniques to connect the evolutionary process to its underlying molecular genetics. This is a thorough and courageous approach to a difficult problem of ultimately understanding the mechanisms that "make a parasite successful". They also explore the specificity versus generality of the evolved responses touching upon another central question in evolutionary biology. All three reviewers are generally positive about the manuscript, and concur on the originality and importance of the approach and of the question posed. However, they also suggest important clarifications, additions and extensions that would benefit the manuscript. A great deal of the proposed changes are mostly directed to improve the reading, the clarify of its data and message, and to deepen some aspects of its conclusions and discussion. Discussion/interpretation Several requests and recommendations have been put forward by reviewers that I summarize as: 1) Reviewer 2 expresses concern with the interpretation of mel SNasr venom composition evolution as it could derive from an experimental design artifact. Please address this concern argumentatively or experimentally; 2) The potential paradox between survival at $100 \%$ (sim and mel strains) and the evolution of venom composition (reviewer \#2) must be addressed; 3 ) The relationship between the success of experimental evolution and the phylogenetic distance of the host used relative to the parasitoid strain; 4) Reviewer \#3 has an interesting suggestion to correlate venom evolution with the two distinct strategies for parasitic success (avoidance and evasion), as well as some mention/discussion of parthenogenesis that you may consider; 5) Another potential relationship to explore and discuss pertains to the success of experimental evolution (replicate extinction) vis-a-vis the phylogenetic distance of the host used relative to the parasitoid strain. Analyses and Format: All reviewers have remarks concerning the presentation of the data that should be revised, mostly to ease the reader's job. 1) Address the issue of infection status classification (reviewer 2\#) 2)

Reviewer \#1 concerns and suggestions (shared by reviewer \#2 and myself) regarding figures 1 and 3 and tables 1 and 2. In addition, please review some of the legends (tables and figures) to ensure all necessary elements are provided to fully understand the information they contain. For example, what is "estimate" on figures S2 and S3, how what is calculated and what does it mean? 3) Justify and explain the use of MANOVA and address the apparent lack of nesting raised by reviewer \#1 and correct the minor points on statistics presentation pointed out by reviewer \#2. 4) I am not sure about this but I wonder if figure S1 should not be part of the main text. I find it really helpful... 5) Please consider the remaining small points included in all three reviews. A final recommendation would be to revise the syntax throughout the manuscript. It need not be Shakespeare but some revision would ease the read. For example, the second term of the first two sentences of the introduction need revision: "and strong selection...success"; "but also host species". ${ }^{* *}$ Additional requirements of the 
managing board**: As indicated in the 'How does it work?' section and in the code of conduct, please make sure that: -Data are available to readers, either in the text or through an open data repository such as Zenodo (free), Dryad or some other institutional repository. Data must be reusable, thus metadata or accompanying text must carefully describe the data. -Details on quantitative analyses (e.g., data treatment and statistical scripts in R, bioinformatic pipeline scripts, etc.) and details concerning simulations (scripts, codes) are available to readers in the text, as appendices, or through an open data repository, such as Zenodo, Dryad or some other institutional repository. The scripts or codes must be carefully described so that they can be reused. -Details on experimental procedures are available to readers in the text or as appendices. -Authors have no financial conflict of interest relating to the article. The article must contain a "Conflict of interest disclosure" paragraph before the reference section containing this sentence: "The authors of this preprint declare that they have no financial conflict of interest with the content of this article." If appropriate, this disclosure may be completed by a sentence indicating that some of the authors are $\mathrm{PCl}$ recommenders: "XXX is one of the $\mathrm{PCI} X X X$ recommenders."

\section{Preprint DOI: https://doi.org/10.1101/2020.10.24.353417}

\section{Reviewed by anonymous reviewer, 2020-11-18 17:05}

Review of the paper: Parasitic success and venom composition evolve upon specialization of parasitoid wasps to different host species

In their manuscript Cavigliasso et al performed an experimental eolutinary experiment by crossing two parasitoid lines with different host range and subsequent rearing of F2 descendants on different hosts. Parasitic success and venom composition were tested at different time points after rearing on different hosts. More specifically parasitic success was assessed as either the capacity to inhibit encapsulation of the capacity to evade capsules. The evolution of venom composition was assessed using 1-d gels and comparison of band intensities as well as statistical analysis of the results. Specific focus was on members of RhoGAPs and of the serpin family.

General comments: In general, I think this is an elegant study of the evolutionary dynamics of a hostparasitoid interaction that is expected to be under strong selection. I am wondering though whether it would be possible to correlate the set of proteins under selection with the initial observation of two different strategies (avoidance of encapsulation in the first place versus evasion from capsules). Are there any proteins that evolved in combinations that affected primarily one of these strategies? Is there any indication whether the two prime candidates (RhoGAPs and serpins) are expected to differentially affect encapsulation?

I also think one might include mention of parthenogenetic wasps. One explanation that has been put forward for a parthenogenetic mode of reproduction in parasitoid wasps (and possible other parasites) is that it allows the fixation of optimal combinations of virulence factors and prevents in an evolutionary landscape the drop into a suboptimal valley due to the combination of incompatibility or less compatibility between virulence factors. Even an asexual mode of reproduction will of course loose its effects in the long term in an ever-changing co-evolutionary race (which may explain the occasional switch to sexual reproduction in some species). My feeling is that some of the F2-host combinations the authors studied including some that were unstable ended up in such evolutionary troughs.

Specific comments: Line 40: in* not on Line 41: subject agreement, is* not are Line 57: here was* Line 67: introduce LbGAP? why did you look into GAP proteins in relation to venom/success? Line 63, 67: delete "indeed," becoming redundant and distracting Line 88: had" Line 102: Originated* Line 130: never allowed to oviposit (parasitize) instead of parasite? Line 147: fix tense Line 168: global analysis: perhaps specify Line 184: redundant citations Line 190: tense Line 193: tense Line 212: In* one host, in* another. Line 410: what previous mass spec data? Line 417: since oxidoreductase was also very abundant why was it left out of subsequent selection analyses? Line 451-457: general experimental set up and aims written so much clearer-something more like this in the intro would be very helpful 


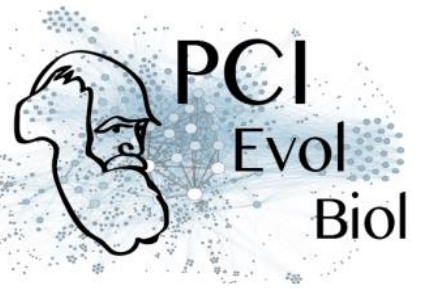

Line 450-601: discussion is perhaps too redundant with results. Perhaps trim down.

Line 503, 522,548: Indeed used quite often. Delete "indeed". Means nothing and is confusing.

Line 517: more information about they there is a cost for virulence factors in one fly over another would be very interesting, see also general points.

Line 544-549: Is there more information about the kind of immune response mounted by drosophila and how the prophenoloxidase cascade may differ in different species and how this might be affect by geography?

Line 578: information about Rho and GAPs and SPN and their involvement and function in parasitization would be nice. As would information about why these proteins are favourable for acting upon in an evolutionary manner.

Line 585: IbSPN mispelled

\section{Reviewed by allexandre leitão, 2020-11-29 10:57}

This study tackles a fascinating question in evolutionary biology, what makes a parasite successful? The biological system studied, Drosophila species as hosts for the parasitoid wasp L.boulardi, is well established and its ecological relevance has been demonstrated. Interestingly, the authors use a similar approach used to demonstrate how Drosophila hosts evolve resistance to parasitoid wasps. This can make future studies very complementary and the further dissection of the system will be aided by recent sequencing of the L.boulardi genome. The article is well written and clear. The predictions are well established and carefully tested.

Essential revisions:

1) The classification of the infection status is confusing. Parasitic success is partitioned in two phenotypes, one where the parasitoid larva is found with no signs of capsule and another one where the wasp larva is found with signs of capsule formation. These phenotypes should be complementary. But, in certain cases, the authors report $100 \%$ of both phenotypes (for example, figure $1 \mathrm{~B}$ at generation $\mathrm{F} 3$ ). How is this possible? It may be my misunderstanding but really struggle to make sense of those numbers and I think any reader would have the same problem.

2) It is somehow surprising to see that the venom composition is selected when wasps are maintained in a very susceptible host like D.melanogaster SNasr. The selective pressure should very reduce to change venom composition. Certainly, the authors put forward an interesting hypothesis to explain this observation, that a cost may be associated with certain venom proteins. However, this change may be an artefact of the crossing scheme used in this study. It is known that crosses with certain strains of L.boulardi results in variable levels of female fertility, in F0 and F1 (Allemand, R. et al., 2002 Ann Soc Entomol, 38(4), pp. 319-332.). If this phenomena occurs asymmetrically between the two cross directions, then at F2 the representation of each genotype can already be biased, given that we are dealing with a haplodiploid system. To exclude this hypothesis, the authors should test female fertility (measuring the sex ratio of the offspring) in the F1 of both cross directions.

Minor points:

1) When possible, report actual $p$ values, do not report things like $p>0.05$ for a single $p$ value.

2) When possible, report the statistical comparisons being made. For example: line 303 "This increase seemed to result solely from the increased capacity to escape from the capsule (Figure 1B, Table S2, GLMM, $p=0.001$ ". What is the comparison reported in here?

3) I would suggest changing the nomenclature of generation to reflect the start of selection. F3, is one generation of selection which can be easily be mixed and interpreted as having 3 generations of selection. 


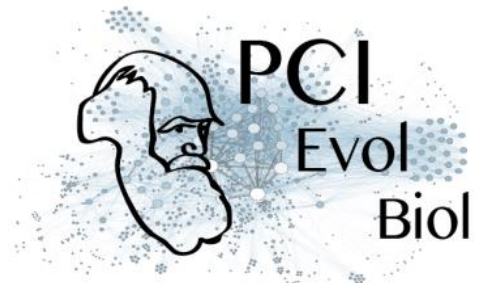

4) The legend for figure $1 \mathrm{C}$ is incorrect. Line 845 "The host listed to the right of each bar plot is the "selection host", those listed down below are the "tested hosts", used for parasitism assays." It should be the other way around.

5) The discussion would benefit with the inclusion of a similar artificial selection study done with another parasitoid wasp species (Kraaijeveld, A. R. et al. (2010), Evolution, 55(9), pp. 1815-1821.)

\section{Reviewed by Simon Fellous, 2020-12-03 11:41}

A. Description This rich manuscript describes the results of an experimental evolution project on the ability of parasitoid to infect different strains of hosts. Analysis is largely rooted in venom composition evolution, in line with previous work by the same research group. The methods are sound and the results prolific. The figures are sometimes complex and hard to grasp, but the writing is very clear.

\section{B. Overall assessment regarding impact and suitability for $\mathrm{PCI}$ Evol Biol}

This is work is at the intercept between evolutionary biology and mechanistic approaches. Indeed, the authors made a great deal of efforts at identifying venom molecules that underlay parasitoid success. This enabled investigating whether venom component had general or specialized effects relative to host strains. The discussion mostly compares current results to those of previous studies with the same species. Implications for general evolutionary theory are therefore scarce in the discussion - a little too scarce in my opinion as an evolutionary biologist. The manuscript may benefit from a more general rooting in the literature, beyond the specific cases of the species (sometimes even strains) of insects used. However, the obvious quality of the work justifies its recommendation once a couple of comments (listed below) have been addressed.

\section{Interpretations and discussion}

There are two elements about which I would welcome a deeper interpretation. 1. About selection on host strains (namely Dmel S and Dsim) that both ancestral parasitoid could infect (and on which the cross had $100 \%$ sucses from the first generation) : there is nonetheless evidence for directional evolution of venom composition. This may seem a paradox (isn't venom here to overcome host defenses?), that the authors solve by evoking unspecified "costs" (e.g. line 489-490). If parasitoids have $100 \%$ infection success form the first generation, that means selection occurred at the adult stage rather than the egg/larval stages. Moreover, the fact that there is differential evolution of the venoms in different susceptible hosts suggests the costs of production of venoms components were host specific. I think these elements should be clarified and argued. 2. L472-479: interesting bit of discussion on survival (and later extinction) of the parasitoids on a host strain the two ancestors could not parasitize. This striking line of reasoning suggests ability to infect relied on codominance rather than the combination of alleles at different loci. This hypothesis may be further explored with the analysis of venom composition at F3 in the 1907 line (before the extinction of the parasitoid lines). Do you have the data? This is particularly important as the final sentence of the MS is about this phenomenon that is otherwise little discussed.

The authors selected the parasitoids on standing genetic variation. This is different from de novo mutation as the phenotypic space and the nature of the trade-offs that can be revealed may differ. These are concepts the MS would probably benefit from citing and making clear. It would help comparing this study to the numerous others on the experimental evolution of parasite specialization (many of which using crosses and standing genetic variation).

D. Presentation of the results:

- Fig 1C: if technically correct, the data could be presented in reader-friendlier way. I'd recommend having a table where host of origin and assayed host are on each axis, but not repeated as is on $\mathrm{X}$ axis. The three generations may be put next to each other, in the same table cell, so one could visualize the evolution of the phenotype in time. Specialization/local adaptation is often studied in an axis/off-axis framework, even if this is not studied as such statistically, using the same graphical representation would help. Also, please put the legend 
for the axes on the figure, not only in the legend. (this later comment is valid for several figures)

- Fig 3: the figure is gorgeous but frankly it is challenging to recognize what is described in the results. Treatments overlap too much for that.

- Table 1: is it necessary to keep all bands for which no significant change is reported? (i.e. blank lines). Maybe can you save space here.

- Table 2: an optional suggestion. Maybe have a colon for each host strain, just as in Table 1. Readers could see at a glance how bands varied together, or not on each host.

E. Stats and clarifications:

- Please clarify why a MANOVA is an appropriate framework to study the evolution of venom composition (i.e. multiple phenotypes). In particular, reader should know how this may reveal qualitative changes in venom composition (one component increases, the other decreases), when MANOVA is often used to study the correlated variation of several responses. Interaction terms can be used - within and between subject interactions - but this is not very clear in the manuscript. Maybe a response to the referee would suffice.

- Unless I missed it, I see no form of nesting of the populations (i.e. replicates) within host strain (or use of a random effect) in the MANOVAS and LDAs. If I am not mistaken, this is necessary to test among host variation taking into account inter-replicate variation, instead of inter-sample variation. How is this taken into account?

- I should also state that I don't have the statistical knowledge to evaluate each of the numerous statistical methods used, some are fairly complex.

F. minor comments:

- Strain availability. You refer to Gif strain numbers. Are these strains available to the community, deposited in stock centers?

- L130: I guess you mean "parasitize", or "oviposit".

- L503: please be more specific. This trade-off only occurred when comparing yakuba 307 and melanogaster $\mathrm{R}$ strains. A reference to the figure would help too. 